\title{
Open
}

\section{Clinical performance of the CytoScan Dx Assay in diagnosing developmental delay/intellectual disability}

\author{
Rolph Pfundt, PhD1, Kat Kwiatkowski, MPH², Alan Roter, PhD², Anju Shukla, MS², \\ Eric Thorland, $\mathrm{PhD}^{3}$, Richard Hockett, $\mathrm{MD}^{4}$, Barbara DuPont, PhD ${ }^{5}$, \\ Eric T. Fung, MD, $\mathrm{PhD}^{2}$ and Alka Chaubey, $\mathrm{PhD}^{5}$
}

\begin{abstract}
Purpose: The prevalence of developmental disabilities in the United States is reported to be $13.87 \%$ across all racial, ethnic, and socioeconomic groups. Microarrays have been recommended as first-tier tests for these patients. This study reports the diagnostic yield and potential actionability of findings using a high-density chromosomal microarray (CMA).
\end{abstract}

Methods: The diagnostic yield of CytoScan Dx Assay in 960 patients was assessed with the Riggs criteria of actionability to evaluate predicted clinical utility.

Results: Eighty-six percent of the subjects were assessed using a microarray as part of historical routine patient care (RPC). The rate of pathogenic findings was similar between RPC (13.3\%) and the CytoScan Dx Assay (13.8\%). Among the 138 patients who did not receive microarray as RPC, the diagnostic yield for CytoScan Dx Assay was $23.9 \%$ as compared with $14.5 \%$, indicating a $9.4 \%$ improvement when using higher-resolution methods. Thirty-five percent of patients with abnormal findings had predicted clinical management implications.

Conclusions: This is the first study to assess the clinical performance of CytoScan Dx Assay. The assay's diagnostic yields are similar to those found in other studies of CMAs. Thirty-five percent of patients with abnormal findings are predicted to have clinical management implications that may improve health outcomes.

Genet Med advance online publication 16 April 2015

Key Words: chromosomal microarray; clinical performance; congenital anomalies; developmental delay; intellectual disability

\section{INTRODUCTION}

The prevalence of developmental disabilities is $13.87 \%$ across all racial, ethnic, and socioeconomic groups, and 1 in 33 babies is born with congenital anomalies in the United States. ${ }^{1,2}$ Frequently, persons with developmental disabilities (developmental delay and/or intellectual disability (DD/ID)) present with one or more congenital anomalies or dysmorphic features. These affected individuals have lifelong challenges, including difficulties with physical movement, learning, and social interaction. Early intervention is important for providing better outcomes for these children with special needs. Despite this, on average, diagnosis of developmental disability in children does not occur until they have reached the age of 4 years, or 2.6 years in children with developmental disability and intellectual disability. ${ }^{3}$ Establishing an underlying diagnosis early has the potential to reduce health-care costs and provide physicians and families with information about the disorder affecting the child, prognosis, and comorbidity, all of which have implications beyond medical treatment.

Historically, with the use of traditional cytogenetic techniques such as karyotyping and fluorescence in situ hybridization (FISH), only a relatively small fraction of patients $(\sim 6 \%)$ were successfully diagnosed, owing to limits on resolution. However, in recent years, with the advent of new techniques such as the chromosomal microarray (CMA), the yield has increased 10-18\%.,5 Additionally, it was recently reported by Shashi et al. ${ }^{6}$ that when a combination of laboratory tools including a microarray was used, $46 \%$ of patients suspected of having a genetic disease were diagnosed and $72 \%$ of these diagnoses were made on the first visit. The authors also showed that a genetic diagnosis in a patient leads to an average cost reduction of more than $\$ 1,435$ in direct genetic laboratory tests (including number of visits to diagnosis and cost of all genetic tests performed) as compared with undiagnosed patients. This is likely a gross underestimate of the true savings because this reports only one portion of typical costs incurred for these patients. It has also been reported that microarray analysis in support of a patient's standard of care impacts patient management up to $70 \%$ of the time. ${ }^{7-10}$ Because clinical management decisions are sometimes subjective, the percentages vary. In two large retrospective studies representing slightly less than a total of 75,000 patients, it was estimated that $35-46 \%$ of patients with pathogenic cytogenetic findings and $7 \%$ of all patients would have some sort of change in clinical management based on microarray results. ${ }^{7.8}$ Two other studies reported on retrospective cohorts for which actual rates of clinical implications were available and found that more than $50 \%$ of all patients with abnormalities had clinical management changes based on microarray results. ${ }^{9,11}$ Although microarrays are now common first-tier tests in this patient population and

${ }^{1}$ Radboud University Medical Center, Nijmegen, The Netherlands; ${ }^{2}$ Affymetrix, Inc., Santa Clara, California, USA; ${ }^{3}$ Mayo Clinic, Rochester, Minnesota, USA; ${ }^{4}$ CombiMatrix Diagnostics, Irvine, California, USA; ${ }^{5}$ Greenwood Genetic Center, Greenwood, South Carolina, USA. Correspondence: Alka Chaubey (alka@ggc.org)

Submitted 23 October 2014; accepted 4 March 2015; advance online publication 16 April 2015. doi:10.1038/gim.2015.51 
supported by medical guidelines from the American College of Medical Genetics and Genomics (ACMG), ${ }^{12}$ the International Collaboration for Clinical Genomics (ISCA/ICCG), ${ }^{5}$ and the American Academy of Neurology, ${ }^{13}$ payer reimbursement for testing is inconsistent, indicating the need for additional systematic studies assessing the changes in patient management that occur as a result of microarray testing.

In a consecutive cohort of 960 patients who had previously undergone standard genetic testing for suspicion of a genetic disorder related to DD/ID, congenital anomalies, and/or dysmorphic features, we assessed the diagnostic yield of the CytoScan Dx Assay as compared with historical patient reports or routine patient care (RPC). To determine how this relates to management implications, we assessed the clinical utility of the CytoScan Dx Assay by applying the Riggs criteria of actionability to the chromosomal abnormalities identified by the CytoScan Dx Assay. In 2013, Riggs et al. ${ }^{8}$ published an article describing 186 phenotypes that were clinically actionable, potentially diagnosable via CMA, and that are causally linked to specific genes and/or chromosomal loci (see Supplementary Table S1 online). They further ranked each phenotype according to the level of evidence available. Clinical actionability was described as requiring at least one of the following recommended interventions for the patient: (i) referral to a specialist, (ii) further diagnostic testing, (iii) surgical/interventional procedure (indicated or contraindicated), (iv) surveillance, (v) medication (prescribed or contraindicated), (vi) lifestyle changes, and (vii) other.

\section{Patient samples}

\section{MATERIALS AND METHODS}

Surplus samples from patients who underwent chromosomal testing were collected from July 2009 to July 2012 at three laboratories: Greenwood Genetic Center (Greenwood, SC), CombiMatrix (San Diego, CA), and the Mayo Clinic (Rochester, $\mathrm{MN})$. Study inclusion criteria were applied to samples as follows: (i) DNA samples from postnatal patients referred for chromosomal analysis based on suspicion of DD/ID, congenital anomalies, and/or dysmorphic features; (ii) prospectively collected or retrospectively banked gDNA (genomic DNA) samples if they were collected from consecutive patients meeting the study criteria during a specified period of time; (iii) sample type was blood-derived gDNA; (iv) gDNA samples extracted from whole blood collected using either ethylenediaminetetraacetic acid or heparin blood collection tubes; (v) gDNA sample concentration was $>150 \mathrm{ng} / \mu \mathrm{l}$ via optical density measurement or $\geq 75 \mathrm{ng} / \mu \mathrm{l}$ via PicoGreen (Life Technologies, Eugene, OR) measurement and diluted in $1 \times$ Molecular Grade TE Buffer (Affymetrix, Santa Clara, CA) pH 8.0; (vi) gDNA sample volume was $\geq 20 \mu \mathrm{l}$; and (vii) gDNA sample had been analyzed using a whole-genome analysis method (e.g., microarray, karyotype) and both the test results and patient diagnostic report could be provided. Samples were excluded if the gDNA sample was analyzed using any Affymetrix microarray as part of RPC to avoid self-comparison of Affymetrix microarrays. This study was approved by the institutional review boards of Greenwood Genetic Center, Mayo Clinic, and CombiMatrix Diagnostics.

\section{Genetic analyses}

Routine patient care (RPC) at these institutions varied and may have comprised one or more copy-number methods such as karyotype, FISH, CMA (excluding any Affymetrix array), polymerase chain reaction, or multiplex ligation-dependent probe amplification. Each institution's qualified cytogeneticist or molecular pathologist generated an overall clinical laboratory interpretation for each sample and also assessed each of the chromosomal copy-number variant $(\mathrm{CNV})$ regions reported in each sample and classified them as benign, pathogenic, or variant of unknown significance (VOUS). Samples were then provided to Affymetrix (Santa Clara, CA) for further analysis. gDNA samples were run at Affymetrix laboratories on the CytoScan Dx Assay, and results were provided to an independent cytogeneticist who generated an overall clinical laboratory interpretation for each sample, including a syndrome diagnosis, if appropriate. The cytogeneticist also assessed each of the sample's CNVs and classified them as benign, pathogenic, or VOUS. The cytogeneticist was permitted to request parental results; if the results were requested and available as part of RPC at the clinical laboratory of origin, then the cytogeneticist was permitted to use them to complete his interpretation. Neither the original clinical laboratory diagnosis nor data from testing conducted during RPC such as karyotyping, FISH, CMA, polymerase chain reaction, multiplex ligation-dependent probe amplification, or any other type of copy-number data were available to the cytogeneticist when interpreting the CytoScan Dx Assay results. All of the clinical and laboratory

Table 1 Clinical interpretation results

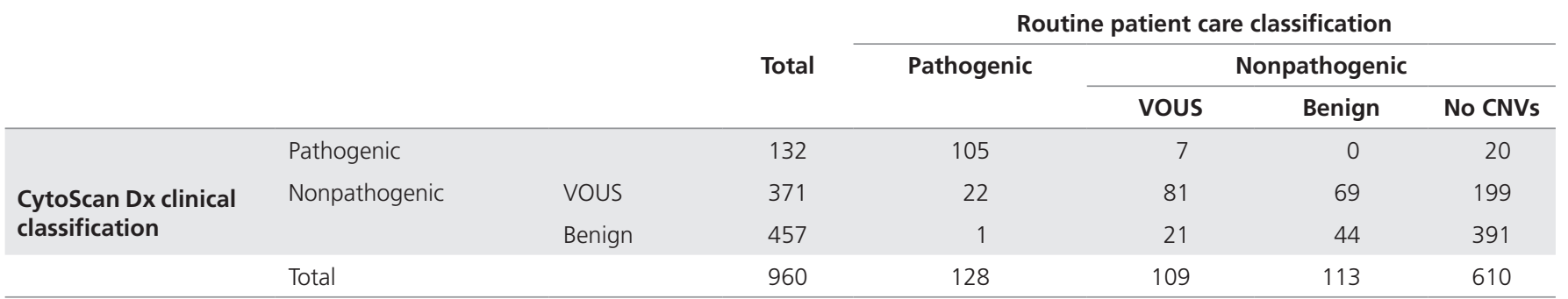

CNV, chromosomal copy-number variant; VOUS, variant of unknown significance. 
data were de-identified prior to entry into DATATRAK EDC (DATATRAK International, Mayfield Heights, $\mathrm{OH}$; version 12.0.0) by the participating institution; monitoring for data quality was conducted by a clinical research organization (Pharm-Olam International, Houston, TX).

\section{Data analysis}

Using Human Genome Build 19 (Genome Reference Consortium GRCh37), CytoScan Dx Assay CNV coordinates were compared with the coordinates of actionable microarray findings listed in the study by Riggs et al. ${ }^{8}$ There were two matching classifications. In the first, if the Riggs region was characterized as a chromosomal region and did not have a specific gene implicated, then the region had to be fully enclosed within a CytoScan Dx Assay CNV region. In the second, if Riggs characterized a specific gene as the region of significance, a match was considered when there was any overlap with a CytoScan Dx Assay CNV (i.e., any overlap would disrupt the gene and function). Rates and descriptive statistics are reported. Calculations were performed using Microsoft Excel 2010.

\section{RESULTS}

A total of 1,171 samples were collected from the three participating sites. Each site confirmed screening of consecutive subjects for inclusion into the study. One hundred fifty-six samples failed to meet inclusion criteria; $96.8 \%$ of the screen failures were due to low sample availability (gDNA sample $\leq 20 \mu \mathrm{l}$ ) or low gDNA concentration and were excluded from the study. Consequently, a total of 1,015 postnatal blood-derived gDNA samples were collected and submitted to Affymetrix. Forty-eight of these samples failed incoming DNA quality-control metrics and were excluded. Therefore, 967 gDNA samples were determined to be eligible for CytoScan Dx Assay. On sample processing, two samples failed array quality-control metrics, one sample was highly segmented $(>1,000$ segments and unable to be loaded into the data-management system) and was not sent for interpretation, and four were excluded by the cytogeneticist as being uninterpretable because of the high number of $\mathrm{CNV}$ segments; therefore, the final analysis data set contained 960 samples. Supplementary Figure $\mathbf{S 1}$ online illustrates the sample disposition and the number of samples included in the analyses.

Site 1 contributed $49.7 \%$ of the samples in the study, and sites 2 and 3 contributing 20.6 and $29.7 \%$, respectively. The average age of subjects was $7.8 \pm 11.3$ years, and $61.7 \%$ were male. Overall, $86 \%$ of the subjects had been previously assessed using a microarray as part of RPC. Four hundred twenty-five CNVs were reported by the investigative sites versus 10,743 identified on CytoScan Dx Assay, which translates into 11.2 $\pm 4.1 \mathrm{CNVs}$ per patient. Sites varied in reporting criteria and did not routinely report every $\mathrm{CNV}$ identified.

\section{Diagnostic yield and absence of heterozygosity}

The diagnostic yield (calculated as the rate of pathogenic findings) was similar between RPC (13.3\% or 128/960) and the CytoScan Dx Assay (13.8\% or 132/960) (Table 1) and 23.9\% or 33/138 (95\% confidence interval, 17.6-31.7\%) as compared with $14.5 \%$ or $20 / 138$ (95\% confidence interval, 9.6-21.3\%) in individuals who did not receive microarray as RPC. These differences were not statistically significant due to the wide confidence interval based on the small sample size of individuals who did not receive a microarray as part of RPC. Absence of heterozygosity $(\mathrm{AOH})$ was reported by the cytogeneticist interpreting CytoScan Dx Assay results in 91 patients. $\mathrm{AOH}$ regions were reported on a variety of chromosomes and had sizes ranging from 5 to $649 \mathrm{Mb}$; the median size was $11.7 \mathrm{Mb}$. Uniparental disomy was identified that predicted a diagnosis of Prader-Willi or Angelman in one sample. However, because $\mathrm{AOH}$ results were not requested from RPC, no comparison is possible. However, obtaining $\mathrm{AOH}$ information in addition to copy-number information from the same platform is known to maximize the diagnostic yield from array testing. ${ }^{14}$

\section{Predicted clinical utility}

Of the patients with pathogenic findings (comprising 58 CNVs) identified by CytoScan Dx Assay, 35\% or 46/132 had predicted clinical management implications. One patient

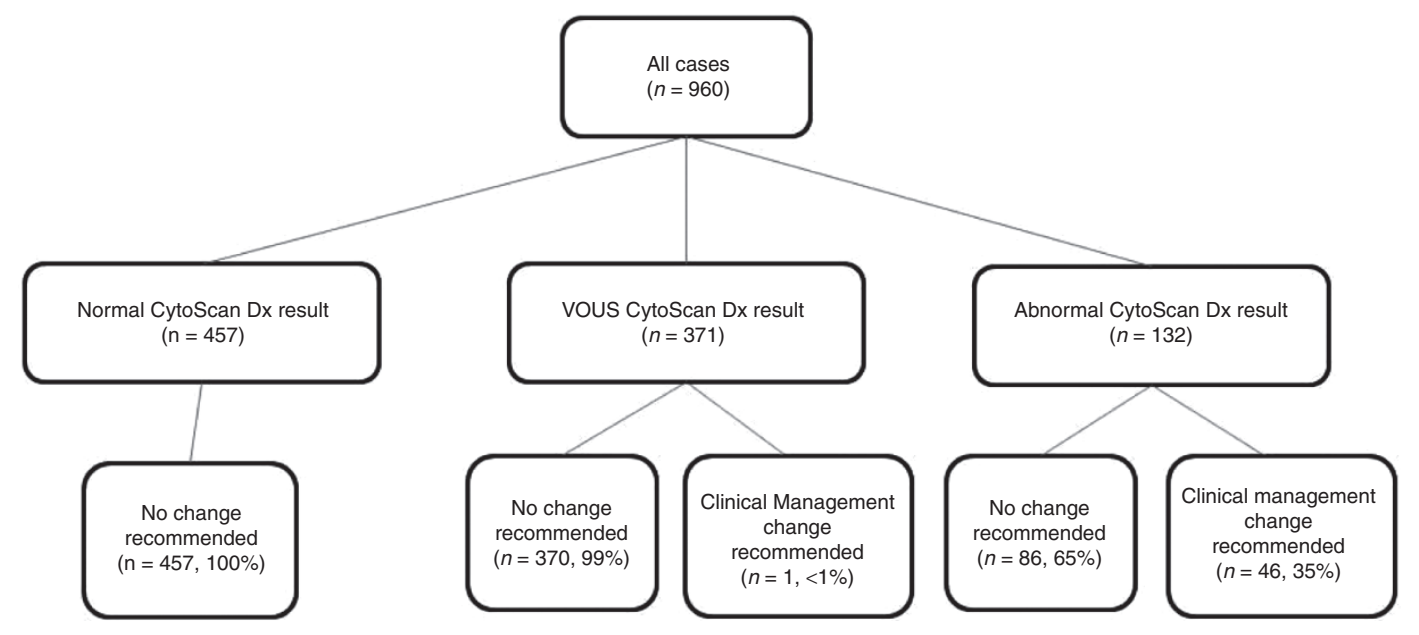

Figure 1 Classification of CytoScan Dx Assay results and predicted clinical management changes. 
Table 2 Description of the patient phenotypes that have clinical management implications

\begin{tabular}{lc}
$\begin{array}{l}\text { Phenotype with documented clinical management } \\
\text { implications }\end{array}$ & $\begin{array}{c}\text { No. of } \\
\text { patients }\end{array}$ \\
\hline 16p13.11 duplication syndrome & 7 \\
\hline 22q11.2-related cardiac phenotype & 5 \\
\hline 1q21.1 deletion syndrome & 4 \\
\hline Familial pulmonary fibrosis & 4 \\
\hline Neurofibromatosis type 1 & 4 \\
\hline Supravalvular aortic stenosis & 3 \\
\hline 16p13.11 microdeletion syndrome & 2 \\
\hline 1q21.1 duplication syndrome & 2 \\
\hline Angelman syndrome & 2 \\
\hline MECP2 duplication syndrome & 2 \\
\hline Steroid sulfatase deficiency; X-linked ichthyosis & 2 \\
\hline 16p11.2 duplication syndrome & 1 \\
\hline 22q11.2 deletion syndrome & 1 \\
\hline 46,XY complete gonadal dysgenesis/disorder of sex & 1 \\
\hline development & 1 \\
\hline Autism spectrum disorders & 1 \\
\hline Charcot-Marie-Tooth type 1a & 1 \\
\hline Duchenne/Becker muscular dystrophy & 1 \\
\hline Holoprosencephaly & 1 \\
\hline Kleefstra syndrome & 1 \\
\hline MAPT-related disorders & 1 \\
\hline Pelizaeus-Merzbacher disease & 1 \\
\hline Phelan-McDermid syndrome & 1 \\
\hline Polycystic kidney disease & 1 \\
\hline PTEN hamartoma tumor syndrome & 1 \\
\hline Retinoblastoma & 1 \\
\hline Smith Magenis & 1 \\
\hline Von-Hippel Lindau & 1 \\
\hline Wolf-Hirschhorn syndrome & 1 \\
\hline
\end{tabular}

MAPT, microtubule-associated protein tau; PTEN, phosphatidylinositol 3,4,5-trisphosphate 3-phosphate and dual-specificity protein phosphatase.

was identified by use of $\mathrm{AOH}$ information with a diagnosis of Prader-Willi/Angelman. Another patient was identified as having a VOUS and was recommended for management changes (Figure 1). The VOUS case was a woman with $\sim 60$ $\mathrm{Kb}$ copy gain (CN state of 3; chr17:15,136,578-15,196,703 [hg19]) of the PMP22 gene (exons 1-4; CCDS11168.1). This copy gain is considerably smaller than the $1.5-\mathrm{Mb}$ classical Charcot-Marie-Tooth type 1a (CMT1A) duplication (chr17:13,968,607-15,434,038[hg19]). However, this finding implicates significant clinical management that would be applicable for CMT1A patients.

Most commonly, 16p13.11 microduplication syndrome was diagnosed, followed by 22.q11.2-related cardiac phenotype. Table 2 provides the phenotypes with documented clinical management implications that were identified in the current study.

The RPC did not routinely report all identified CNVs, so a comparison with the predicted clinical management changes using the Riggs criteria identified by CytoScan Dx Assay is not possible.

CytoScan Dx Assay identified 20 additional abnormal CNVs in historical patient reports, $25 \%$ of which had predicted clinical management implications (Table 3 ).

To illustrate the clinical utility of CytoScan Dx Assay, one of the patients was a 20-month-old boy of African-American ethnicity who presented in the clinic for the evaluation of DD, hypotonia, low weight, and microcephaly. There were no perinatal complications, although he had been born at $36^{1 / 2}$ weeks. Previous magnetic resonance imaging yielded normal results. Family history revealed that he had three older half-siblings, one of whom had a history of attention-deficit hyperactivity disorder and another who had history of heart surgery for a heart murmur. Physical examination at the time of evaluation showed a weight of $9.9 \mathrm{~kg}$ (second percentile), height of $80.5 \mathrm{~cm}$ (15th percentile), and head circumference of $44.5 \mathrm{~cm}$ (less than second percentile). He sat at approximately 12 months of age and made some nonspecific sounds (babbling). This individual was very social and interactive. He had no major dysmorphic features (except a flat nasal bridge), and the cardiovascular examination results were noncontributory. However, neurological examination revealed mild truncal hypotonia. RPC genetic testing included karyotyping, methylation studies for Prader-Willi/Angelman syndrome, fragile- $\mathrm{X}$ testing, and myotonic dystrophy testing, all of which had normal results. CytoScan Dx Assay identified a deletion of 7q11.23, which is consistent with a clinical diagnosis of Williams-Beuren syndrome (WBS). WBS is a contiguous gene deletion syndrome in patients presenting with ID, hypercalcemia, hypercalciuria, hypothyroidism, failure to thrive in infancy, supravalvar aortic stenosis, distinctive facial features, stellate iris, hoarse voice, hernia, rectal prolapse, and joint limitation or laxity. The general recommendations for management of this disorder call for aggressive therapies and screening. Ophthalmologic evaluation, kidney ultrasound, thyroid testing, and total and ionized calcium level testing are some routine evaluations that are recommended, demonstrating the complexity in clinical management of WBS.

Several microdeletion/microduplication syndromes were identified by CytoScan Dx (and missed by RPC) in this study, including (but not limited to) 3q29 microduplication, 22q11 microduplication, $16 \mathrm{p} 11.2$ microduplication, $16 \mathrm{p} 11.2$ microdeletion, KBG syndrome, and Floating-Harbor syndrome. Some of these cases would have warranted significant clinical management at the time of diagnosis, whereas for others finding a genetic cause would have ended the diagnostic odyssey. However, because the RPC deemed these cases normal, no other follow-up information was provided by the respective sites for this study.

\section{DISCUSSION}

The current study was conducted to assess the clinical performance of CytoScan Dx Assay in patients with DD, ID, and/or congenital anomalies who were referred for whole-genome 
Table 3 Additional CNVs identified by CytoScan Dx Assay

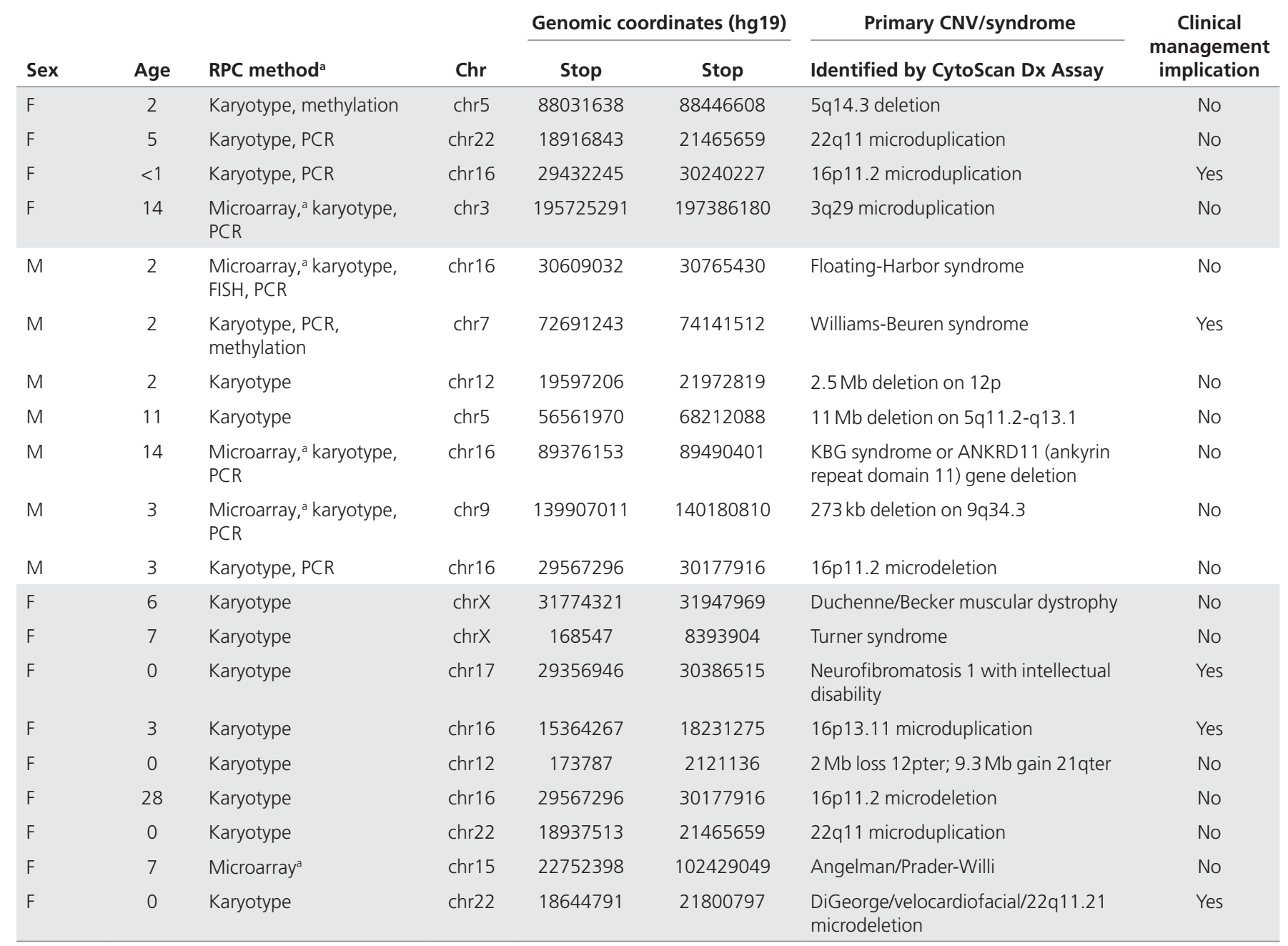

CNV, chromosomal copy-number variant; FISH, fluorescence in situ hybridization; PCR, polymerase chain reaction; RPC, routine patient care.

aNon-Affymetrix Microarray.

chromosomal testing. CytoScan Dx Assay identified rare pathogenic CNVs in $13.8 \%$ of these patients. This diagnostic yield is comparable to previous reports of historical RPC, which often used microarrays to assess these patients, and it is also consistent with the published literature on diagnostic yield in this patient population. ${ }^{4,5,15}$ This study was originally designed to meet US Food and Drug Administration requirements and the interpreting cytogeneticist did not have access to any complementary information, as is standard in laboratory analysis (i.e., clinical data or supplementary laboratory testing). To obtain a more realistic view of CytoScan Dx Assay's diagnostic yield, it may be useful to reanalyze this data set with the complete laboratory information available to a qualified cytogeneticist.

Importantly, this study illustrates that one-third of patients with an abnormal chromosomal anomaly identified by CytoScan Dx Assay have predicted clinical management changes with levels of evidence of 1-3. Riggs et al. reported that $46 \%$ of all pathogenic or likely pathogenic CNVs submitted to their database had management implications with levels of evidence of 1 or 2 . This difference in percentage could be due to the fact that their cohort was not a consecutive series but, rather, a freeze of a database to which many laboratories submit array results. Level 1 evidence is described as practice guidelines endorsed by a professional society, level 2 evidence is peer-reviewed publications available for making medical management recommendations, and level 3 evidence indicates that there are no relevant peer-reviewed publications but potential management implications may be warranted based on clinical judgment (see Riggs et al. ${ }^{8}$ for a full description of the evidentiary levels). The current study did not have a category for "likely pathogenic" as the Riggs cohort did, and the cytogeneticist interpreting the results of this study did so in isolation, i.e., did not have access to any clinical information about these patients and very little data regarding parental results. Nonetheless, even if we were to remove CNVs with a level of evidence of 3 for management implications, only six patients would be removed from the list of clinically actionable subjects, which means that this consecutive cohort still 
predicts that $30 \%(40 / 132)$ of pathogenic CNVs would necessitate clinical management changes.

It is now commonly known that high-resolution wholegenome CMAs such as CytoScan Dx Assay have the ability to identify CNVs that may be missed by other technologies such as karyotyping, FISH, and targeted or lower-resolution array platforms due to inadequate coverage and/or lower resolution. Finding these CNVs can end the diagnostic odyssey for patients and families, potentially reduce health-care costs by avoiding unnecessary testing, provide additional information to inform reproductive health for family planning, and substantially affect patient care. In this cohort, CytoScan Dx Assay identified an additional $20 \mathrm{CNVs}$ compared with the RPC results, which typically used one or more methods of chromosomal analysis; $25 \%$ of these additional CNVs would result in predicted clinical management changes.

There are several limitations of our study. The comparison of the diagnostic yield of RPC with that of CytoScan Dx Assay is confounded by the fact that laboratories that interpreted the historical RPC results may have had access to complementary laboratory results and parental data, whereas the cytogeneticist interpreting the results from CytoScan Dx Assay did not have access to clinical or accompanying laboratory information and access to parental testing was extremely limited. Another limitation is that the patient cohort reported here was not necessarily evaluated with microarrays as a firsttier test. Many of the known, actionable CNVs are larger and based on lower-resolution platforms. Over the past 3-5 years, high-resolution platforms have identified many small CNVs that underlie ID/DD disorders and therefore may lead to increases in diagnostic yield and the actionable rate for some of these patients. ${ }^{16}$ Finally, the comparison with the Riggs criteria is predictive in nature and not a reflection of actual practice. Future studies should be prospective and follow patients for actual clinical management changes.

\section{Conclusion}

In conclusion, this is the first study to assess the clinical performance of CytoScan Dx Assay in a consecutive cohort of patients with DD, ID, and/or congenital anomalies who were referred for whole-genome chromosomal testing. CytoScan Dx Assay's diagnostic yields are similar to those reported in previous studies, with 13.8 and $35 \%$ of patients with pathogenic CNVs identified by CytoScan Dx Assay predicted to have clinical management implications that may improve health outcomes.

\section{SUPPLEMENTARY MATERIAL}

Supplementary material is linked to the online version of the paper at http://www.nature.com/gim

\section{ACKNOWLEDGMENT}

This study was funded by Affymetrix Inc.

\section{DISCLOSURE}

K.K., A.R., A.S., and E.T.F. are salaried employees of Affymetrix. The other authors declare no conflict of interest.

\section{REFERENCES}

1. Boyle CA, Boulet S, Schieve LA, et al. Trends in the prevalence of developmental disabilities in US children, 1997-2008. Pediatrics 2011;127:1034-1042.

2. Heron M, Hoyert DL, Murphy SL, Xu J, Kochanek KD, Tejada-Vera B. Deaths: final data for 2006. Nat/ Vital Stat Rep 2009;57:1-134.

3. Mann JR, Crawford S, Wilson L, McDermott S. Does race influence age of diagnosis for children with developmental delay? Disabil Health J 2008;1: 157-162.

4. Sagoo GS, Butterworth AS, Sanderson S, Shaw-Smith C, Higgins JP, Burton H. Array $\mathrm{CGH}$ in patients with learning disability (mental retardation) and congenital anomalies: updated systematic review and meta-analysis of 19 studies and 13,926 subjects. Genet Med 2009;11:139-146.

5. Miller DT, Adam MP, Aradhya S, et al. Consensus statement: chromosomal microarray is a first-tier clinical diagnostic test for individuals with developmental disabilities or congenital anomalies. Am J Hum Genet 2010;86:749-764.

6. Shashi V, McConkie-Rosell A, Rosell B, et al. The utility of the traditional medical genetics diagnostic evaluation in the context of next-generation sequencing for undiagnosed genetic disorders. Genet Med 2014;16:176-182.

7. Ellison JW, Ravnan JB, Rosenfeld JA, et al. Clinical utility of chromosomal microarray analysis. Pediatrics 2012;130:e1085-e1095.

8. Riggs ER, Wain KE, Riethmaier D, et al. Chromosomal microarray impacts clinical management. Clin Genet 2014;85:147-153.

9. Coulter ME, Miller DT, Harris DJ, et al. Chromosomal microarray testing influences medical management. Genet Med 2011;13:770-776.

10. Saam J, Gudgeon J, Aston E, Brothman AR. How physicians use array comparative genomic hybridization results to guide patient management in children with developmental delay. Genet Med 2008;10:181-186.

11. Henderson LB, Applegate CD, Wohler E, Sheridan MB, Hoover-Fong J, Batista DA. The impact of chromosomal microarray on clinical management: a retrospective analysis. Genet Med 2014;16:657-664.

12. Manning M, Hudgins L; Professional Practice and Guidelines Committee. Array-based technology and recommendations for utilization in medical genetics practice for detection of chromosomal abnormalities. Genet Med 2010;12:742-745

13. American Academy of Neurology. Genetic and metabolic testing in children with global developmental delay. https://www.aan.com/guidelines/home/ getguidelinecontent/489. 2011. Accessed on 6th April 2015

14. Wiszniewska J, Bi W, Shaw C, et al. Combined array CGH plus SNP genome analyses in a single assay for optimized clinical testing. Eur J Hum Genet 2014;22:79-87.

15. Hochstenbach R, van Binsbergen E, Engelen J, et al. Array analysis and karyotyping: workflow consequences based on a retrospective study of 36,325 patients with idiopathic developmental delay in the Netherlands. Eur J Med Genet 2009;52:161-169.

16. Nevado J, Mergener R, Palomares-Bralo M, et al. New microdeletion and microduplication syndromes: A comprehensive review. Genet Mol Biol 2014;37(suppl 1):210-219.

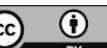

This work is licensed under a Creative Commons Attribution 4.0 International License. The images or other third party material in this article are included in the article's Creative Commons license, unless indicated otherwise in the credit line; if the material is not included under the Creative Commons license, users will need to obtain permission from the license holder to reproduce the material. To view a copy of this license, visit http://creativecommons.org/licenses/by/4.0/ 